\title{
Research Paper \\ Comparing the Structural Standards of Nursing Homes in Markazi Province, Iran With International Standards
}

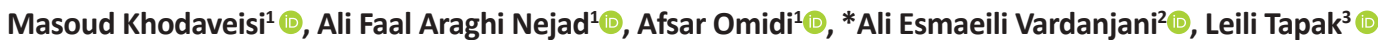

1. Chronic Diseases (Home Care) Research Center, Department of Community Health Nursing, School of Nursing \& Midwifery, Hamadan University of Medical Sciences, Hamadan, Iran.

2. Department of Critical Care Nursing \& Management, School of Nursing and Midwifery, Tehran University of Medical Sciences, Tehran, Iran

3. Department of Biostatistics and Epidemiology, School of Health, Hamadan University of Medical Sciences, Hamadan, Iran.

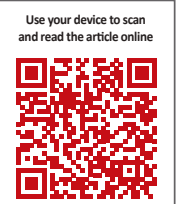

Citation: Khodaveisi M, Faal Araghi Nejad A, Omidi A, Vardanjani Esmaeili A, Tapak L. [Comparing the Structural Standards of Nursing Homes in Markazi Province, Iran With International Standards (Persian)]. Iranian Journal of Ageing. 2018; 13(3):362-371. https://doi.org/10.32598/SIJA.13.3.362

https://doi.org/10.32598/SIJA.13.3.362

(c) (1) (3)

Funding: See Page 370

Received: 10 Apr 2018 Accepted: 22 Jul 2018

Keywords: Nursing Home, Nursing standards Structural standards, Environmental standards, Nursing staff standards

\section{A B S T R A C T}

Objectives In the recent years, the elderly pollution has increased; therefore, the availability of centers that meet their physical and emotional needs has become extremely important. Standardization is one of the approaches to improve the quality of these centers. The purpose of this study was to compare the status of structural standards in nursing homes with international standards.

Methods \& Materials In this descriptive, cross-sectional study, three nursing homes in Markazi Province were studied in 2017. To investigate the status of structural standards, a researcher-made checklist was used, which consisted of the staff standards, physical environment standards, and equipment standards. The validity of this checklist was confirmed using expert panel and its reliability was confirmed by parallel forms reliability. The collected data were analyzed by SPSS 23.

Results Overall, the centers were in moderate situation in terms of structural standards with average score of $83.29 \%$ (mean: 674 [out of 810]). The scores of Imam Javad Nursing home, Ebrahimabad Nursing home, and Meisam Nursing home were $88.40 \%, 88.64 \%$, and $72.84 \%$ (mean: 716,718 , and 590 ), respectively. Conclusion Considering that the structural condition of nursing homes in Markazi Province is lower than international standards, nursing home officials are recommended to improve the quality of their centers to achieve the levels of international standards, especially structural standards.

\section{Extended Abstract}

\section{Objectives}

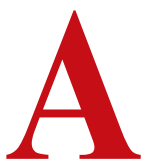

ccording to the World Health Organization, older people are those aged 60 years and over, and their population has grown significantly in recent years [1]. Considering the physical and mental conditions of the elderly, the need for nursing homes is essential. Therefore, ensuring the quality of care in these centers is very important. One of the most effective methods for improving the quality of nursing centers, especially nursing homes, is the use of standards [2]. Standards are descriptions of the status and desirable level of performance based on which quality can be judged [3]. Based on Donabedian model, to determine the quality of health care centers, three standard categories are needed: "structure," "process," and "outcomes." In this regard, this study aimed to investigate the struc-

\section{* Corresponding Author}

\section{Ali Esmaeili Vardanjani, PhD Student}

Address: Department of Critical Care Nursing \& Management, School of Nursing and Midwifery, Tehran University of Medical Sciences, Tehran, Iran.

Tel: +98 (913) 0688603

E-mail: aliesmailiv@yahoo.com 
tural quality of nursing homes in Markazi Province of Iran and its comparison with international standards.

\section{Methods and Materials}

In this descriptive cross-sectional study, all nursing homes in Markazi Province (three centers in Arak city and Ebrahim Abad village) were selected by total enumerative method. The data collection tool was a researcher-made checklist. This study was conducted in three stages with one-month interval. In the first step, the checklist was created by reviewing published papers on the structural standards of the developed countries [5-8]. The final version of the checklist contains 162 items in three dimensions of manpower ( $\mathrm{n}=33)$, physical space $(\mathrm{n}=99)$, and supplies and equipment $(\mathrm{n}=30)$. It was based on 5-point scale from $1=$ non-observed to $5=$ completely observed where the lowest possible score was 162 and the highest 810 .

The quality scores were divided into three levels of weak (162-635), moderate (635-730), and good (735$810)$. In the second stage, the validity and reliability of the checklist were investigated and the face validity was determined by 12 faculty members of the Nursing and Midwifery at Hamadan University of Medical Sciences. Parallel forms of the test were used to measure the reliability of the checklist. In this method, the nursing homes were examined by two observers using the checklist, and then the correlation between their observations was examined. In the third step, the data were collected by using the checklist, and then the collected data were analyzed using descriptive statistics such as mean and standard deviation and performing inferential statistics of Spearman and Pearson correlation tests in SPSS V. 23.

\section{Results}

Results showed that the study nursing homes had met $82.29 \%$ of the standards and achieved an average score of 674 out of a total score of 810 . The Ebrahim Abad Nursing home earned $88.64 \%$ of scores (718 points), Iman Jawad Nursing home $88.40 \%$ (716 points), and Meysam
Nursing home $72.84 \%$ (590 points). In terms of manpower, Ebrahim Abad Nursing home ranked first with $88.67 \%$ followed by Iman Jawad (87.33\%) and Meysam nursing homes $(83.33 \%)$. In terms of physical space, Iman Jawad Nursing home ranked first with $88.69 \%$ followed by Ebrahim Abad (88.28\%) and Meysam nursing homes (69.68\%) and finally, with respect to supplies and equipment, Ebrahim Abad Nursing home ranked first with $89.70 \%$ followed by Iman Jawad (88.48\%) and Meysam nursing homes $(75.76 \%)$ (Table 1$)$.

The results indicated that all study nursing homes were at poor level in terms of using skilled manpower, and although the number of caregivers was at the optimal level, the number of nursing staff was lower than the standard level. The strength of nursing homes was training during work and at the beginning of staffing which was in the correct and standard manner in accordance with the existing rules.

Regarding the physical space standards, nursing homes did not have a favorable status in terms of safety, especially in the corridors and flooring, although they had obtained a satisfactory score. Also, the results showed that the most of study nursing homes had hospital-like design. This can have a direct negative effect on the privacy and independency of the elderly; and since one of the basic structural standards in nursing homes is the resemblance of the nursing homes to a home, it can be said that it has not been met. In terms of supply and equipment, the nursing homes earned acceptable scores in the field of welfare equipment, but in the field of healthcare tools, they failed to achieve acceptable scores, because most of the nursing homes were only well-equipped for normal healthcare supply.

\section{Conclusion}

Standard structural quality of nursing homes investigated in this study was at moderate level. Due to the situation and conditions of the residents of these centers, these centers must be improved, because indepen-

Table 1. Structural qualities of the study nursing homes.

\begin{tabular}{ccccccccc}
\hline \multirow{2}{*}{ Nursing Homes } & \multicolumn{2}{c}{ Manpower } & \multicolumn{2}{c}{ Physical Space } & \multicolumn{2}{c}{ Supplies and Equipment } & \multicolumn{2}{c}{ Total } \\
\cline { 2 - 9 } & Score & $\%$ & Score & $\%$ & Score & $\%$ & Score & $\%$ \\
\hline Ebrahim Abad & 133 & 88.76 & 438 & 88.28 & 148 & 89.70 & 718 & 88.64 \\
Iman Javad & 131 & 87.33 & 439 & 88.69 & 146 & 88.48 & 716 & 88.40 \\
Meisam & 125 & 83.33 & 340 & 68.69 & 125 & 75.76 & 590 & 72.84 \\
\hline & & & & & & & &
\end{tabular}


dency and security and having an active life are among the main rights of the elderly. Moreover, it is necessary to increase the adaptability of the elderly by improving the quality of nursing homes and bringing them closer to international standards and eliminating existing gaps. The existence of rules and standards consistent with international standards for the quality of care and structure of nursing homes can help improve the conditions of the nursing homes and its elderly residents in Iran. So the authorities should work on drafting laws and regulations, especially structural standards.

\section{Ethical Considerations}

\section{Compliance with ethical guidelines}

This study has been approved by the Ethics Committee of Hamadan University of Medical Sciences (code: IR.UMSHA.REC.1395.589).

\section{Funding}

This research was extracted from the second author's thesis in Chronic Diseases (Home Care) Research Center, Department of Community Health Nursing, School of Nursing \& Midwifery, Hamadan University of Medical Sciences, Hamadan, Iran.

\section{Conflict of interest}

All authors certify that this manuscript has neither been published in whole nor in part nor being considered for publication elsewhere. The authors declare no conflict of interest.

\section{Acknowledgements}

Hereby, we appreciate all the people who helped us in conducting this paper. 


\title{
مقايسه استانداردهاى ساختارى خانههاى سالمندان استان مركزى با استانداردهاى بينالمللى

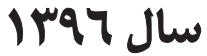

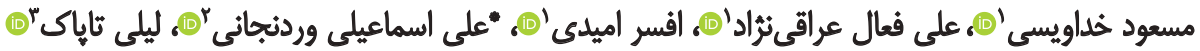

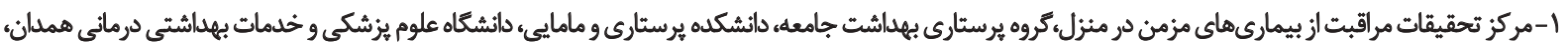 \\ همدان، ايران.

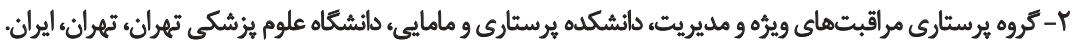

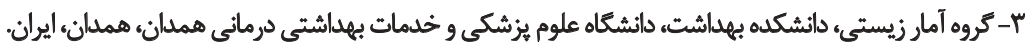

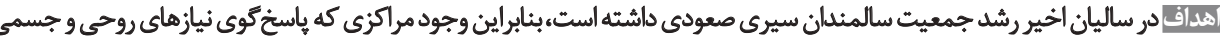

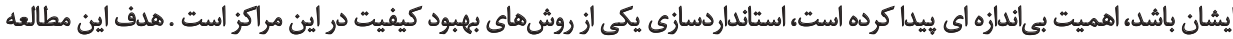

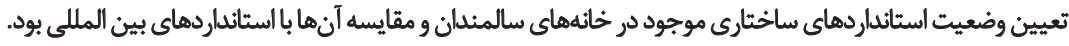

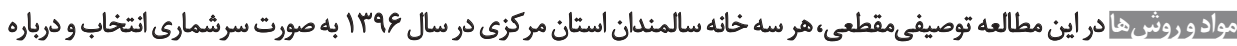

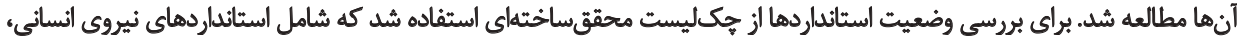

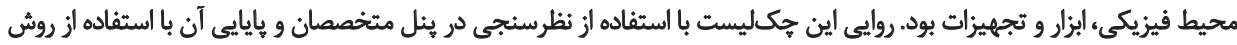

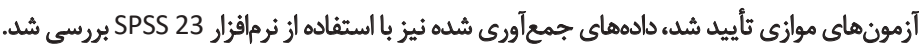

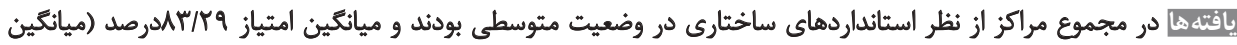
جواد أزVF

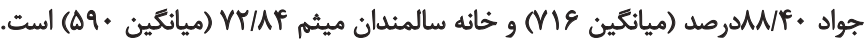

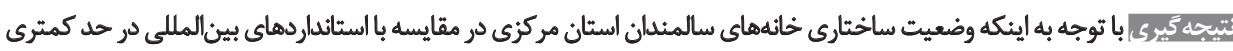

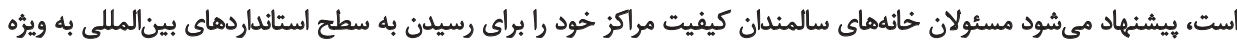

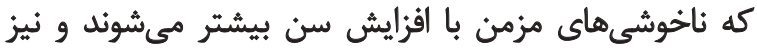

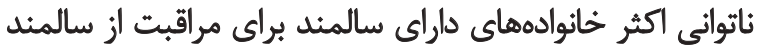

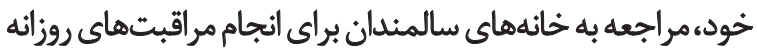

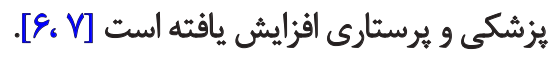

سازمان بهداشت جهاني اقرادى راكه بيشتر از •ع سال دارند،

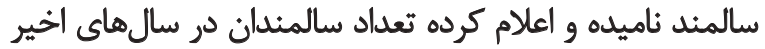

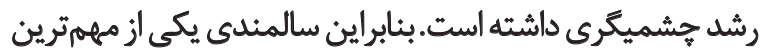

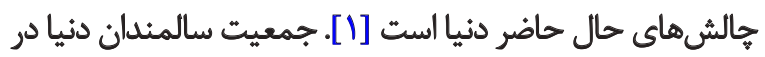

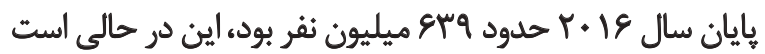
مطالعات نشان مى دهد استفاده از خانههاى سالمندان در ايران

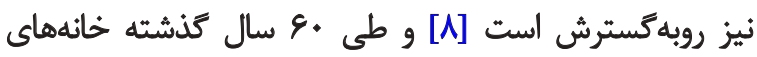

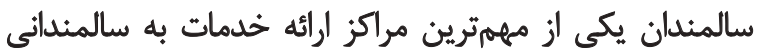

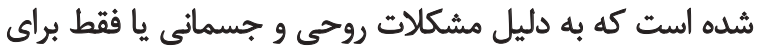

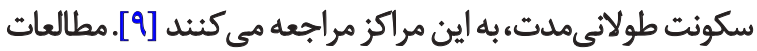

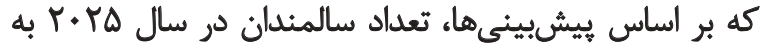

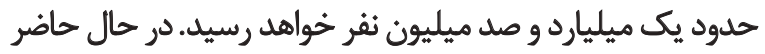

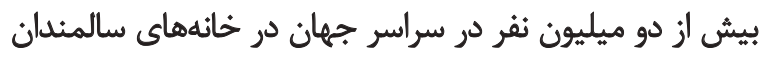

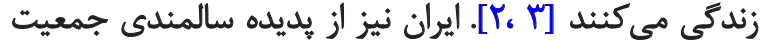

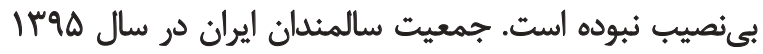

- 
نتايج مطالعه نوردين" و همكاران كه با هدف تعيين وضعيت

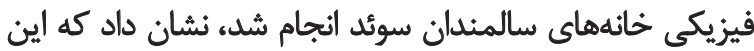

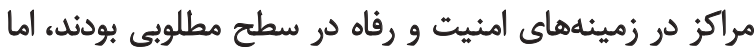

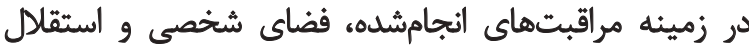

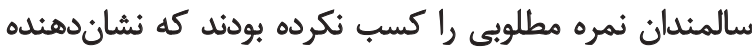

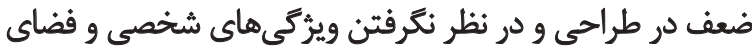

$$
\text { خصوصى سالمندان است [11]]. }
$$

نتايج مطالعه كاثلر و و همكاران كه با هدف بررسي وضعيت

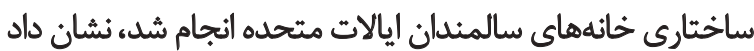

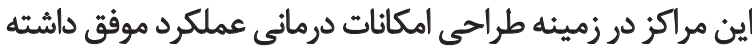

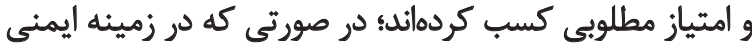

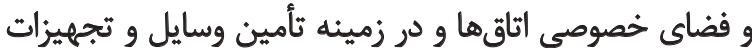

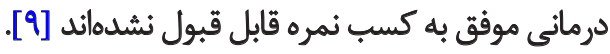
با در نظر كرفتن تأثير مستقيم وضعيث ساختارى خانههاى

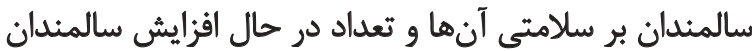

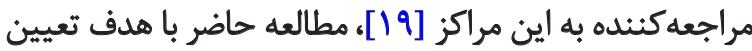
وضعيت ساختارى خانههاى سالمندان استان مركزى و مقائن إنايسه

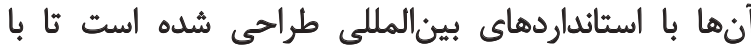

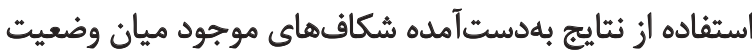

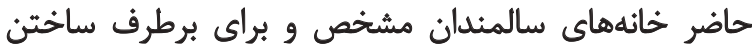

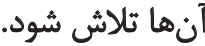

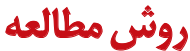

در اين مطالعه توصيفىمقطعى تمامى خانههاى سالمندان

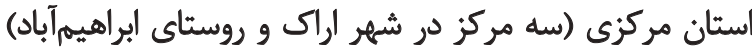

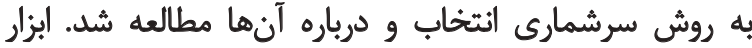

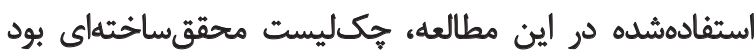

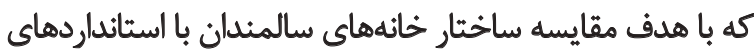

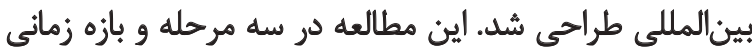

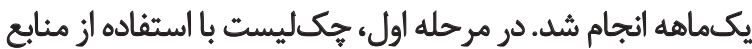

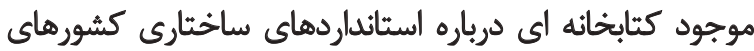

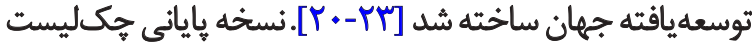

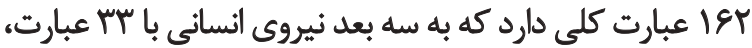

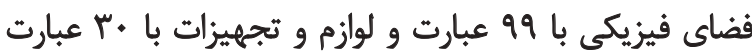

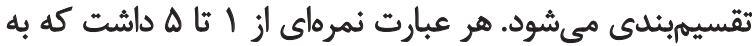

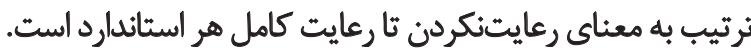

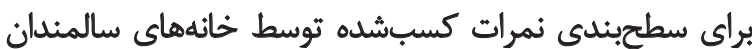

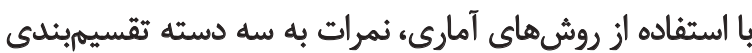

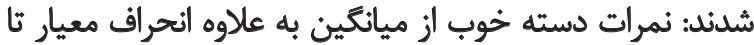

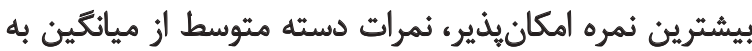

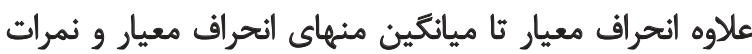

\section{Nordin}

4. Cutler
نشان مي دهند حدود • r درصد از سالمندان ارويايى در خائههاي

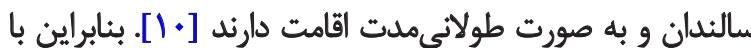

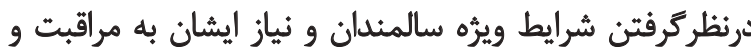

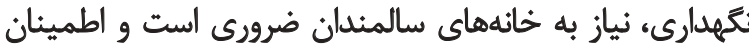

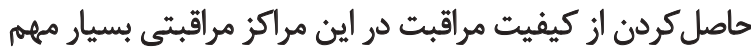

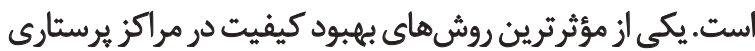

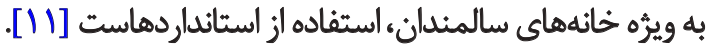
استائداردها، توصيف وضعيت و سطح مطلوب عملكردها

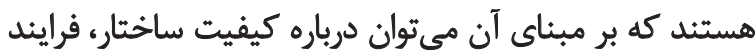

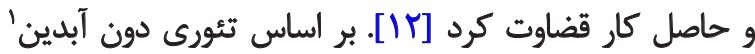

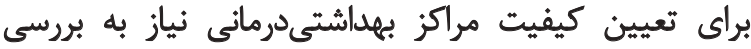

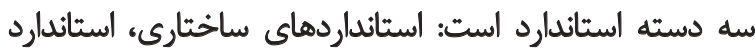

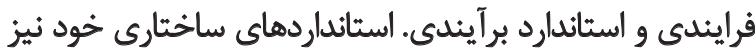

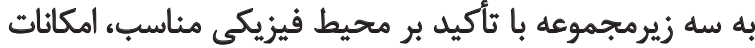

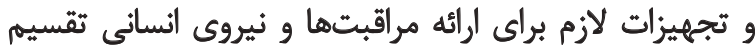

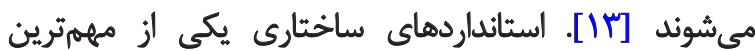

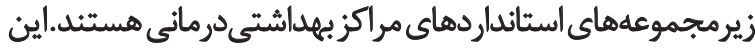

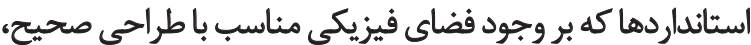

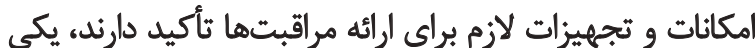

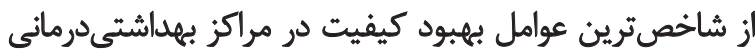

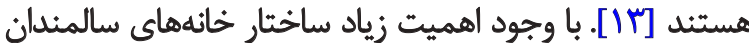

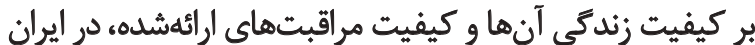

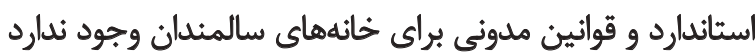

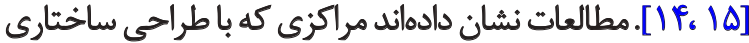

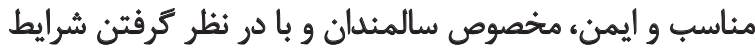

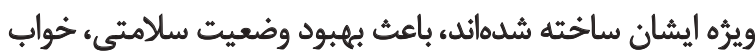
و استقلال در سالمندان مىشوند [19]

نتايج مطالعه اورى و و همكاران كه با هدف بررسى وضعيت

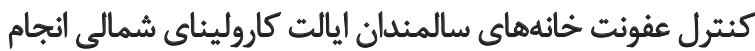

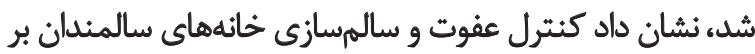

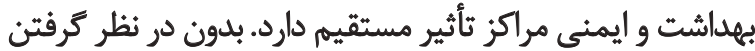

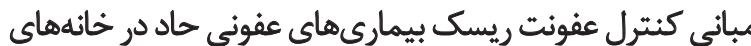

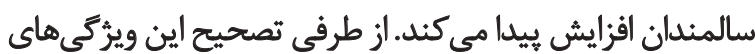

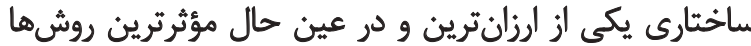

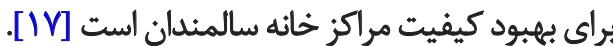
نتايج مطالعه نصيرى و همكاران كه با هدف تعيين وضعيت

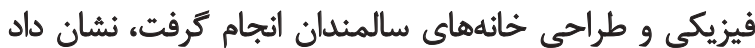

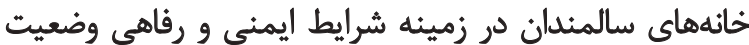

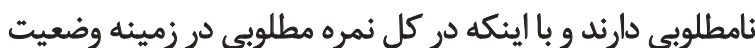

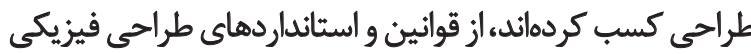

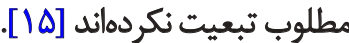

1. Donabedian

2. Avery 


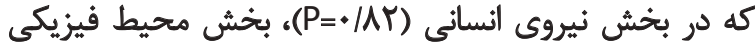
(P=॰/99)

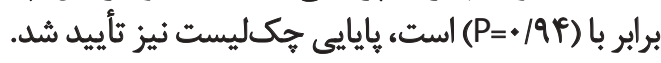

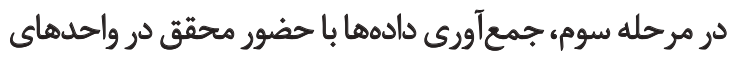

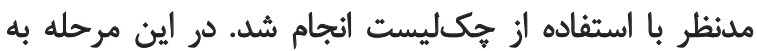

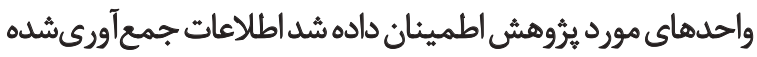

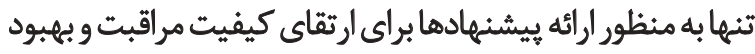

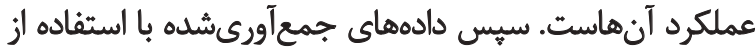

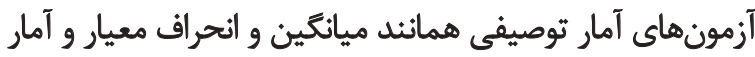

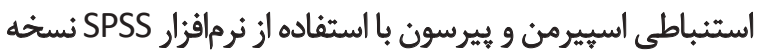

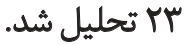

يافتهها

نتايج مطالعه درباره وضعيت استانداردهاى ساختارى خانهاي

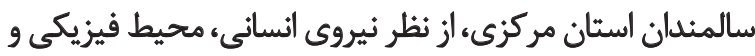

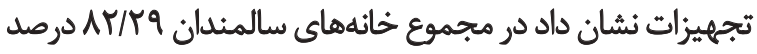
استانداردها را رعايت و ميانكين

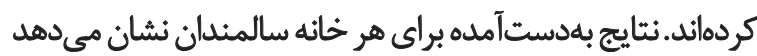

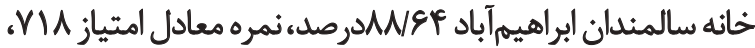

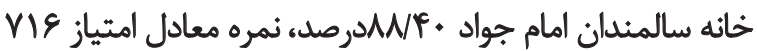

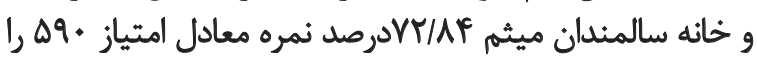
كسب كر دهانل.

در زمينه وضعيت ابعاد استانداردهاي ساختارى خانهائ سالمندان از نظر بعد نيروى انسانى خائه سالمندان ابراهيمارئ آبادياد

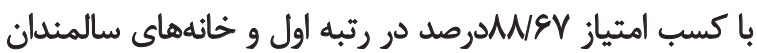

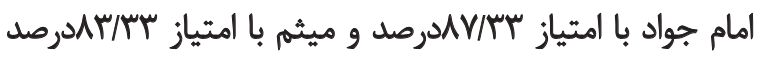

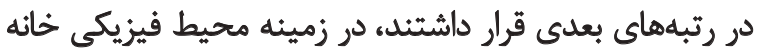

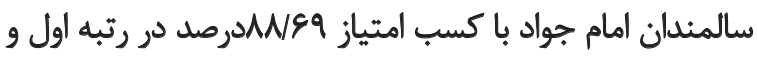

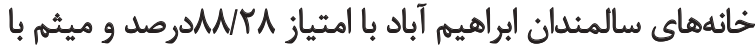

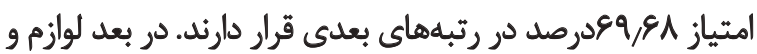

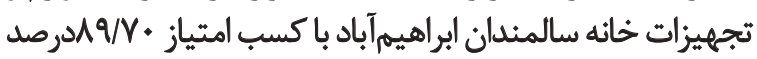

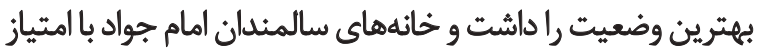

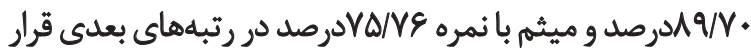

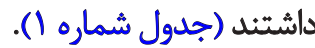

نمرات كسبشده در وضعيت زيرمجموعه هاى ابعاد

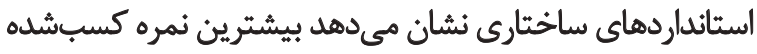

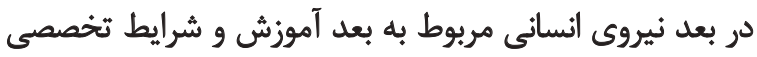

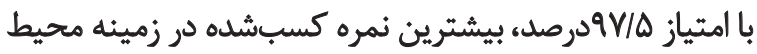

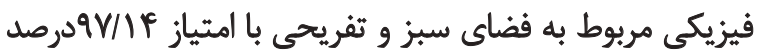

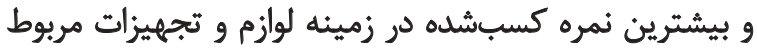

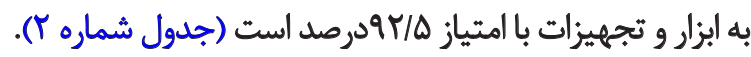

دسته ضعيف از ميانكين منهاى انحراف معيار تا كمترين نمره

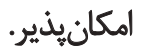

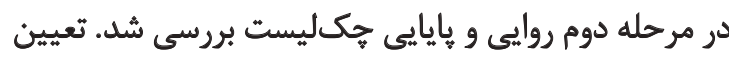

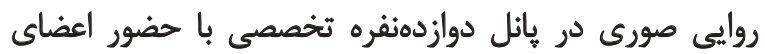

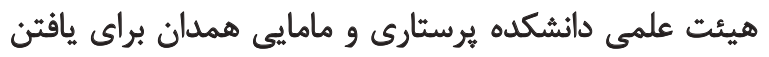

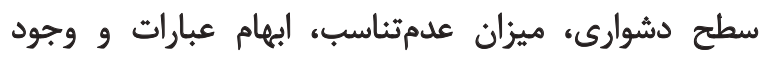

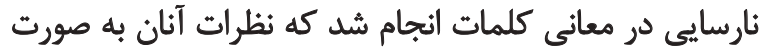

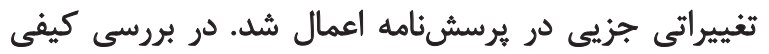

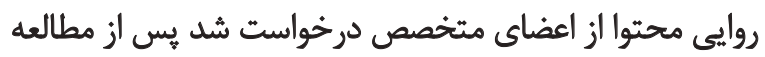

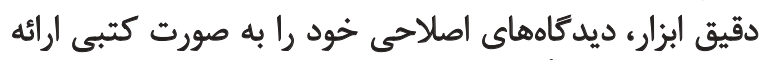

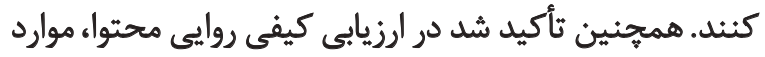

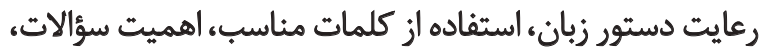

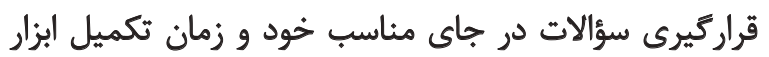

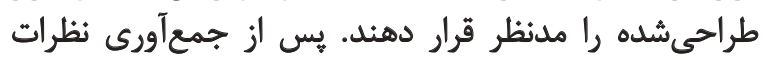

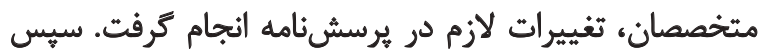

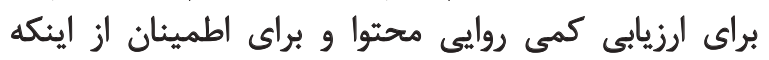

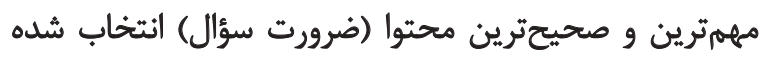

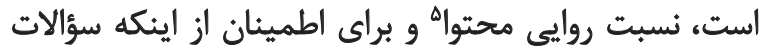

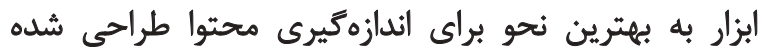

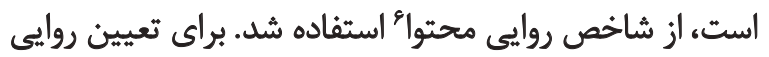

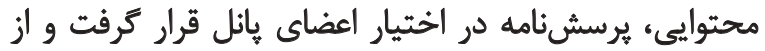

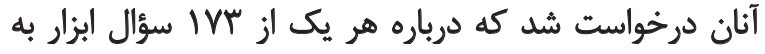

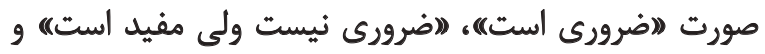

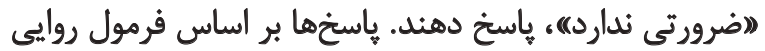

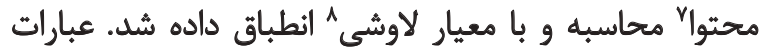

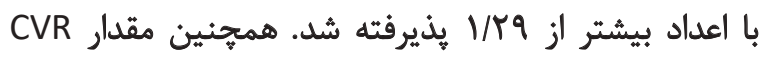

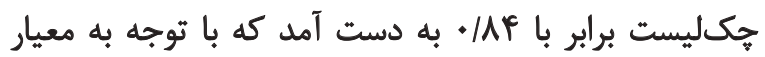

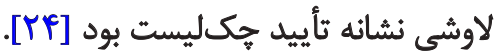

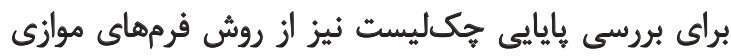

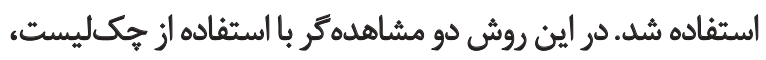

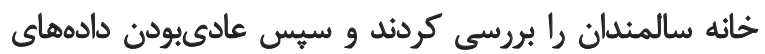

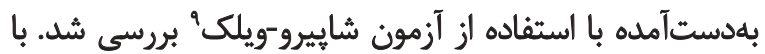

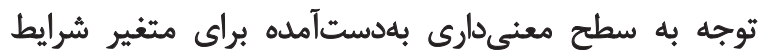

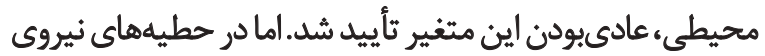

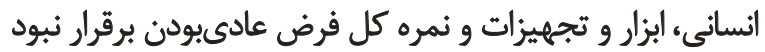

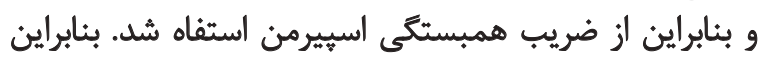

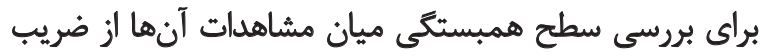

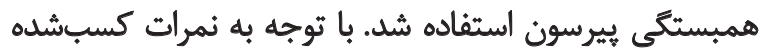

\footnotetext{
5. Ratio validity Content

6. Index validity Content

7. Content Validity Ratio (CVR)

8. Lawshe

9. Shapiro-Wilk
} 
جدول ا. وضعيت استائداردهاي ساختارى خائهاي سالمندان استان مركزى به تفكيك ابعاد آن

\begin{tabular}{|c|c|c|c|c|c|c|c|c|}
\hline \multicolumn{2}{|c|}{ مجموع } & \multicolumn{2}{|c|}{ لوازم و تجهيزات } & \multicolumn{2}{|c|}{ محيط فيزيكى } & \multicolumn{2}{|c|}{ نيروى انسانى } & \multirow{2}{*}{ واهد } \\
\hline ورصد & امتياز & مeرe & امتياز & مرصد & امتياز & مر & امتياز & \\
\hline WEP & nis & $\Delta q / \mathrm{N}$. & IPA & WTA & PrA & WEV & int & ابراهيميم آباد \\
\hline MF. & ne & WFA & life & WEq & prq & AV/MT & $|r|$ & امام ججواد \\
\hline$V T / A F$ & $\Delta q$. & VANG & ITA & ENEA & $M$ & NH/M & ITA & ميثم \\
\hline
\end{tabular}

年

نيروى يرستارى ضعيف عمل كرده بودند. دليل اين ضعف عملكرد

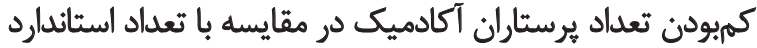

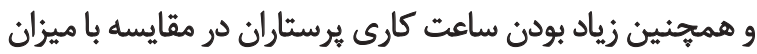

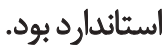

نتايج بهدست آمده نشاندهنده اين بود كه خانههاي سالمندان

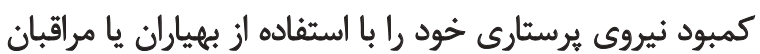

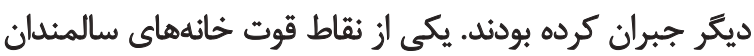

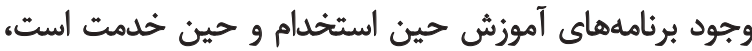

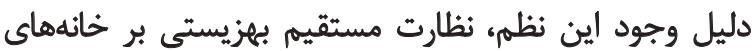

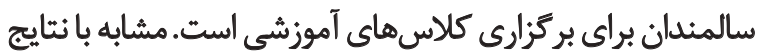

نتايج بهدستآمده درباره استانداردهاي ساختارى خانههاي

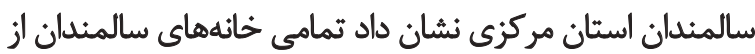

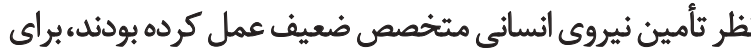

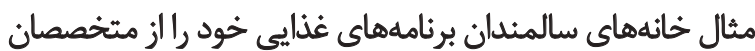

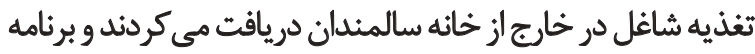

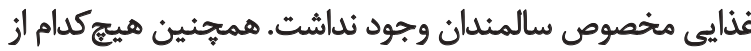
خانهايى سالمندان بررسى شده، مسئول بهداشت داشت دائمى نداشتيند

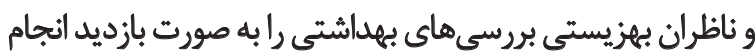

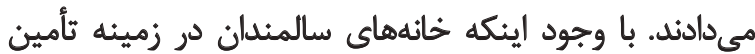

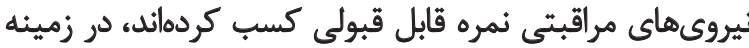

جدول r. وضعيت استانداردهاي ساختارى به تفكيك شاخص هاى تعريفشده در زير مجموعههاي خانه سالمئدان

\begin{tabular}{|c|c|c|c|c|}
\hline ميثم & امام جواد & ابراهيه|آباد & زيرمجموعه & ويرُكى كلى \\
\hline $9 V / \Delta$ & 9. & $9 \Delta$ & أموزش و شرايط تخصصى & \multirow{2}{*}{ نيروى انسائي خانههائ } \\
\hline VNIA & Ne/Re & Ne/NE & تقسيم كار نيروى أسانى & \\
\hline VT/Re & $9 \pi / \Delta \Delta$ & $\Lambda \otimes / A 1$ & التاقها & \multirow{9}{*}{ محيط فيزيكي خانهانهاى } \\
\hline ه. & AD & $\lambda F / M T$ & درها، هاركييكَها، راهروها & \\
\hline as & ar & Af & سيستم كرمايشى و برقر سانى & \\
\hline$n / M$ & WA9 & WA9 & حمام و توالتها & \\
\hline$\Delta \Lambda$ & Af & 94 & رختشويخانه و انتاق خدمه & \\
\hline A. & ivpo & QY/Q & آشيزخانه و غناخورى & \\
\hline AN/AF & $9+/ W$ & Q⿻/ar & ايستكاه برستارى و اثتاق درمان & \\
\hline ro & A. & v. & أببارى و محل نكهباري ابزار خاص & \\
\hline$A T / N E$ & qV/If & $9 V /$ If & فضاى تفريحى & \\
\hline QTH & $91 / 11$ & $A Y / M T$ & ابزار فردى و شخصىى & \multirow{2}{*}{ لوازم و تجهيزات خانيهذاي } \\
\hline$A F / / Y$ & AV/D & $9 T / \Delta$ & ابزار و تجهيزات درمانى & \\
\hline
\end{tabular}

L 
در اين مراكز بر بازدهي خدمات درمانى بيشتر از ايجاد فضايى بيى براني

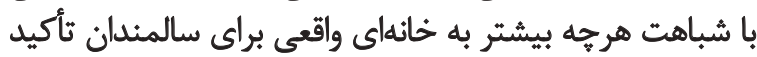

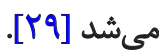

نتايج مطالعه در زمينه وضعيت سالن غذاخورى و آشيرخانه

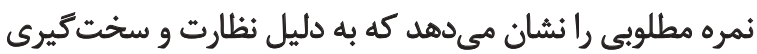

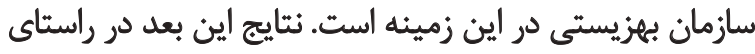

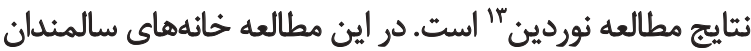

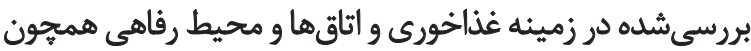

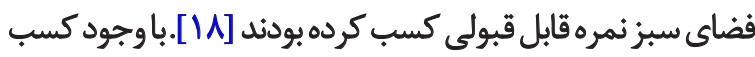

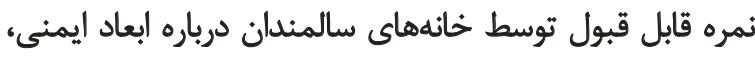

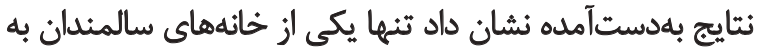

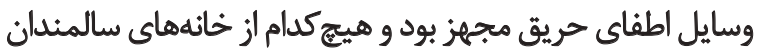
به سيستم اطفا حريق مركزى مجهز نبودند.

در مطالعه ما بعد لوازم و تجهيزات درمانى يكى از ضعيفترين

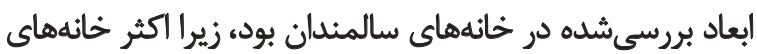

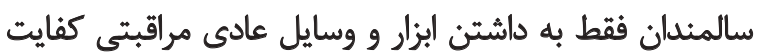

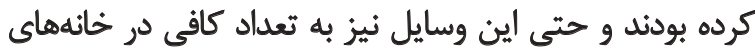

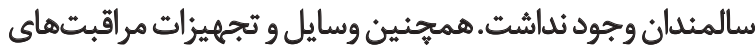

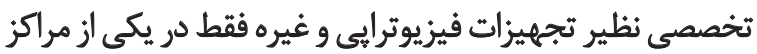

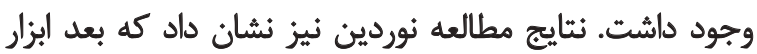

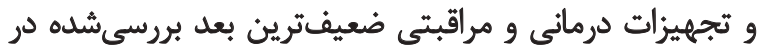

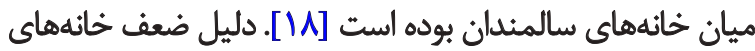

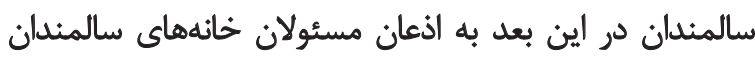

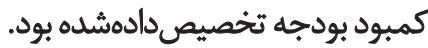

\section{نتيجه تيرىنهايى}

يافتههاى اين مطالعه نشان داد وضعيت استانداردهاى

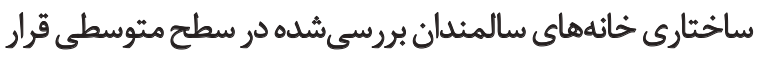

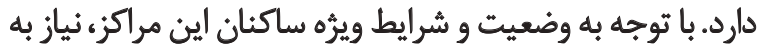

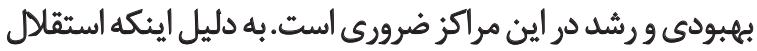

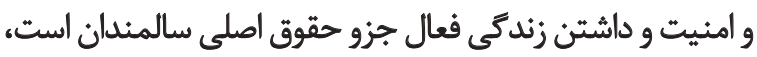

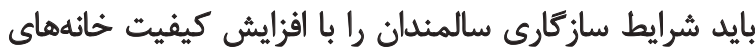

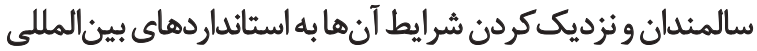

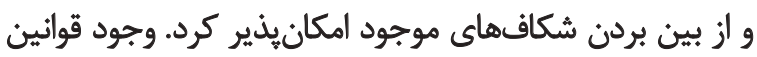

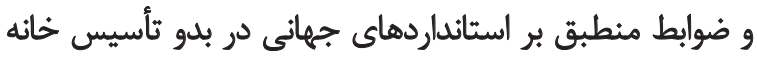

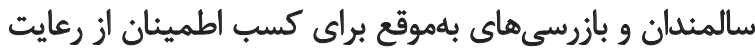

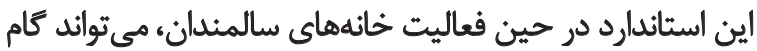

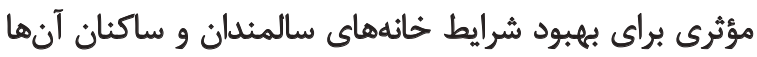

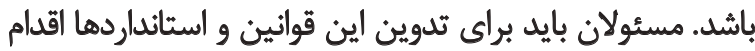

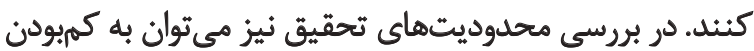
تعداد خانههاى سالمندان نمونه اشاره كرد.

13. Nordin
اين مطالعه، نتايج مطالعه هارينكتون "و همكار بارنش كه در سال

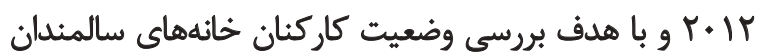

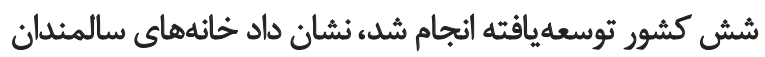

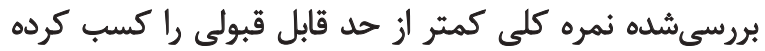

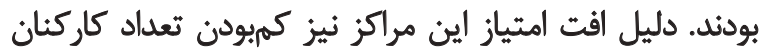

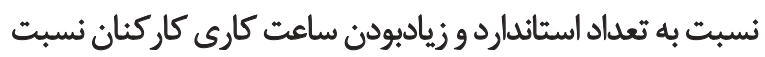

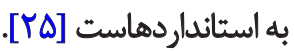

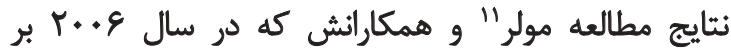

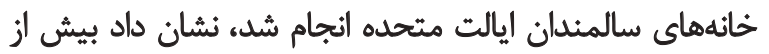

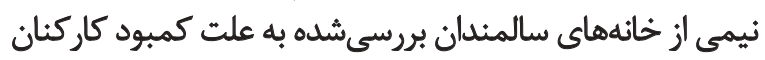

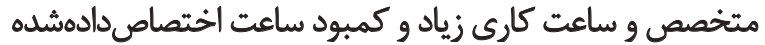

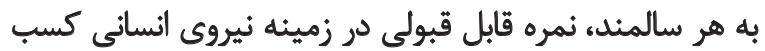

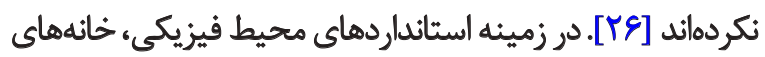

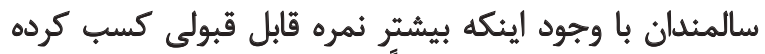

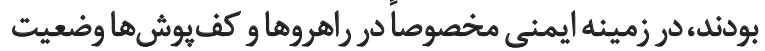

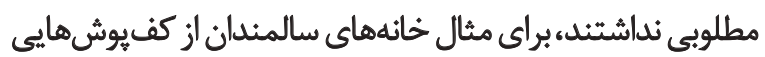

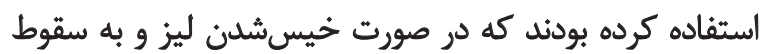

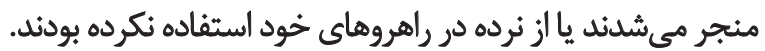

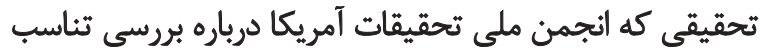

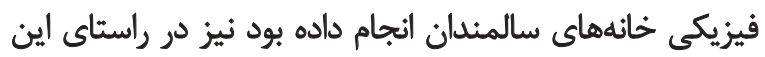

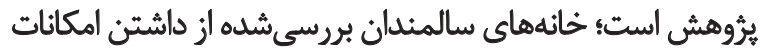

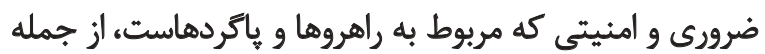
سطح شيبدار مناسب، نرده راهروها و ميله محافظ ئراتردها،

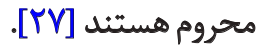

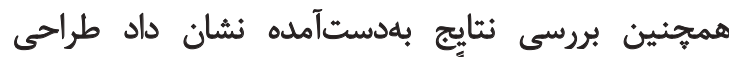

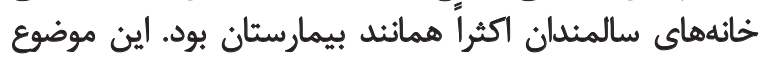

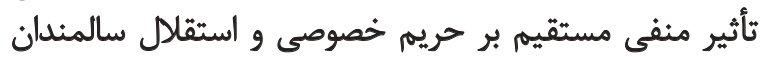

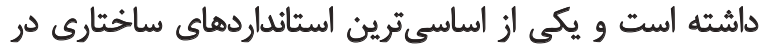

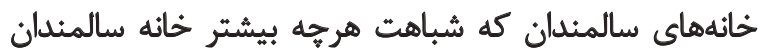

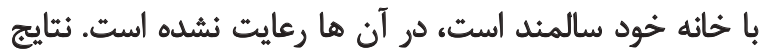

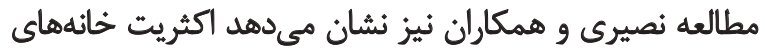

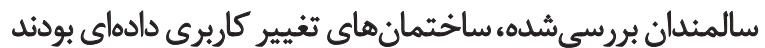

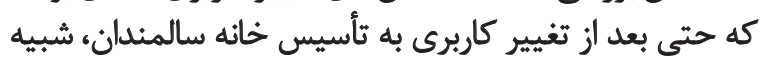

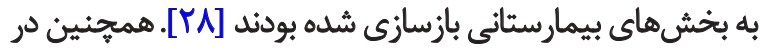

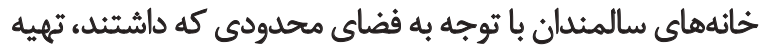

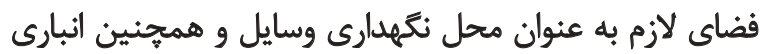

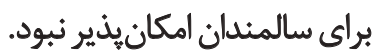

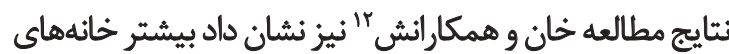

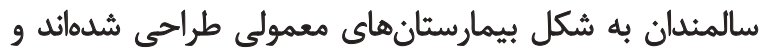

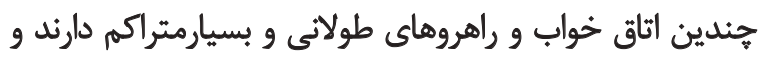


بيشنهادهاي نويسندكان براي مطالعات آينده عبارتند از: 1. با توجه به تعداد كم مطالعات انجامشده درباري نياره استانداردهاي

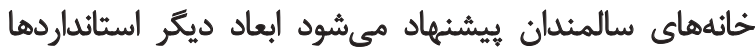

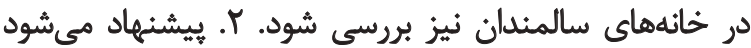

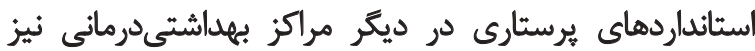

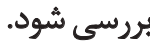

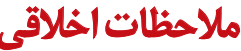
بيروى أز اصول اخلاق يثروهش

اين مطالعه كواهىنامه كميته اخلاق بزشكى را به شماره

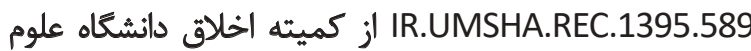
يزشكى همدان كسب كرده است.

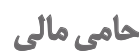
اين مقاله از خاياننامه على فعال عراقى نثراد، گروه يرستارى

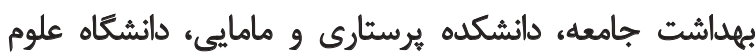
يزشكى و خدمات بهداشتى درمانى همدان ترفته شده است

$$
\text { تعارض منافع }
$$

بنا بر اظهار نظر نويسندكان مسئول در اين مقاله تعارض منافع

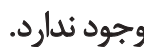

$$
\text { تشكير و قدرواني }
$$

نويسندكان از مسئولان و استادان محترم دانشكاه علوم يزشكى ئري

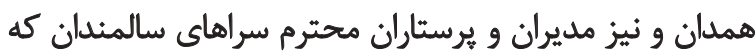
در اين تحقيق ما را يارى كردند، كمال تشكر و قدردان مانى ران را دارند. 


\section{References}

[1] Kinsella KG, Phillips DR. Global aging: The challenge of success. Washington, DC: Population Reference Bureau; 2005.

[2] Abedi H, Lali M, Keyvanniya S, Nikbakht A. [Life experiences of older people who are used to spend their time in parks (Persian)]. Journal of Qualitative Research in Health Sciences. 2013; 2(2):184-93.

[3] The World Bank. Population ages 65 and above [Internet]. 2016 [cited 2017 Apr 09]. Available from: https://data.worldbank. org/indicator/SP.POP.65UP.TO.ZS

[4] Taghdisi MH, Doshmangir P, Dehdari T, Doshmangir L. [The factors affecting healthy lifestyle from elderly vision: A qualitative study (Persian)]. Iranian Journal of Ageing. 2012; 7(27):47-58.

[5] Statistical Center of Iran. [Population of the country in terms of sex in urban and rural areas by province (Persian)] [Internet]. 2016 [Cited 2017 July 28]. Available from: https://www.amar. org.ir/Portals/0/census/1395/results/tables/jamiat/kolli/1koli-jamiat.xls

[6] Darvishpoor Kakhki A, Abed Saeedi J, Delavar A. [Diseases of old people referring to elderly centers of Tehran (Persian)]. Journal of Payavard-e Salamat. 2014; 7(6):479-89.

[7] Salarvand S, Abedi HA. [Causes and motivations of elderly home residency from residents' point of view (Persian)]. Feyz. 2008; 12(2):55-61.

[8] Ghazi K, Foroughan M, Hosseini M, Hosseinzadeh S, Askari $\mathrm{M}$. [The client satisfaction of delivered services in private nursing homes for elderly: a survey in the provinces of Golestan, Mazandaran, Semnan and Northern Khorasan in 2012 (Persian)]. Journal of Sabzevar University of Medical Sciences. 2013; 20(3):320-30.

[9] Cutler LJ, Kane RA, Degenholtz HB, Miller MJ, Grant L. Assessing and comparing physical environments for nursing home residents: Using new tools for greater research specificity. The Gerontologist Journal. 2006; 46(1):42-51. [DOI:10.1093/gerf ont/46.1.42]

[10] Onder G, Carpenter I, Finne-Soveri H, Gindin J, Frijters D, Henrard JC, et al. Assessment of nursing home residents in Europe: The services and health for elderly in long term care (Shelter) study. BMC Health Services Research. 2012; 12(1):5-14. [DOI:10.1186/1472-6963-12-5] [PMID] [PMCID]

[11] Rolland Y, Mathieu C, Piau C, Cayla F, BougetC, Vellas B, et al. Improving the quality of care of long-stay nursing home residents in France. Journal of the American Geriatrics Society. 2016; 64(1):193-9. [DOI:10.1111/jgs.13874] [PMID]

[12] Douglass LM. The effective nurse: Leader and manager. Missouri: Mosby Pub; 1996.

[13] Jafari GA, Khalifegari S, Danaei Kh, Dolotshahi P, Ramezani M, Rohparvar R, et al. [Hospital Accraditation standards in iran (Persian)]. Tehran: Seda Publishers; 2010.

[14] Zarghami E, Sharghi A, Olfat M. [Environmental features affecting the quality of life among elderly residents in nursing homes, Case study: Shemiranat Area in Tehran Province (Persian)]. Journal of Iranian Architecture Studies. 2015; 1(7):111-26.

[15] Nasiri M, Foroughan M, Rashedi V, Makarem A, Jafari Mourjan $\mathrm{B}$. [Compliance to universal design criteria in nursing homes of Tehran (Persian)]. Iranian Journal of Ageing. 2016; 11(2):340-7. [DOI:10.21859/sija-1102340]

[16] Nordin S, McKee K, Wijk H, Elf M. Exploring environmental variation in residential care facilities for older people. Health Environments Research \& Design Journal. 2017; 10(2):49-65. [DOI:10.1177/1937586716648703] [PMID]

[17] Avery C, Humphrey C, Balany JA. Evaluation of five years of nursing home inspection forms: Structural and hygiene-related violation trends. Journal of Environmental Health. 2014; 77(4):14 9. [PMID]

[18] Nordin S. The quality of the physical environment and its association with activities and well-being among older people in residential care facilities [PhD thesis]. Karolinska Institutet, Stockholm, Sweden; 2016.

[19] Abdollahi F, Mohammadpour RA. [Health related quality of life among the elderly living in nursing home and homes (Persian)]. Journal of Mazandaran University of Medical Sciences. 2013; 23(104):20-5.

[20] Masoudi R, Vardanjani E, Ali S, Rabiei L, Moghadassi J, Khayri F, Rahimi Madiseh M. A group-foundation exercise schedule on quality of life and well-being in older men and women. Indian Journal of Science and Technology. 2012; 5(2):2165-9.

[21] Nursing Home Standards Workgroup. Enhanced Nursing Home Standards. Singapore: Ministry of Health; 2014.

[22] Ministery for Health \& Social Services. National minimum standards for care homes for older people. Cardiff: Ministery for Health \& Social Services; 2005.

[23] Office Long Term Care. Rules and Regulations for Nursing Homes: Regulations of Long Term Care. Little Rock, Arkansas Arkansas Department of Human Services; 2006.

[24] LawsheCH. Aquantitative approach tocontent validity.Personnel Psychology. 1975; 28(4):563-75. [DOI:10.1111/j.1744-6570.1975. tb01393.x]

[25] Harrington C, Choiniere J, Goldmann M, Jacobsen FF, Lloyd L, McGregor M, et al. Nursing home staffing standards and staffing levels in six countries. Journal of Nursing Scholarship. 2012 44(1):88-98. [DOI:10.1111/j.1547-5069.2011.01430.x] [PMID]

[26] Mueller C, Arling G, Kane R, Bershadsky J, Holland D, Joy A. Nursing home staffing standards: Their relationship to nurse staffing levels. The Gerontologist. 2006; 46(1):74-80. [DOI:10.1093/ geront/46.1.74] [PMID]

[27] Crews DE, Zavotka S. Aging, disability and frality: Implications for universal design. Journal of Physiological Anthropology. 2006; 25(1):113-8. [DOI:10.2114/jpa2.25.113]

[28] Naseri M, Sadati L, Jalali A, Firuzabadi M, Golchini E. [Assess of the status of the Karaj operating rooms in comparison with international standards in 2011 (Persian)]. Alborz University of Medical Sciences Journal. 2012; 1(4):213-9.

[29] Kane RA, Kane RL, Bershadsky B, Cutler LJ, Giles K, Liu JJ, et al. Measures, indicators, and improvement of quality of life in nursing homes. Report to Centers for Medicare and Medicaid Services. Minneapolis, Minnesota: University of Minnesota; 2004. 